\title{
Interaction between Metal Packages and the Enclosed MMICs
}

\author{
Th. Bolz*', Stu. Member, IEEE, B. Neuhaus*, Stu. Member, IEEE and A. Beyer*, Fellow, IEEE \\ * Duisburg-Essen University, Campus Duisburg, Department of Electrical Engineering and \\ Information Techniques, Bismarckstr. 81, D-47048 Duisburg, Germany \\ ${ }^{\dagger}$ IMST, Carl-Friedrich-Gauss-Str. 2, D-47475 Kamp-Lintfort, Germany \\ E-mail: bolz@imst.de
}

\begin{abstract}
In this paper an inhomogenously filled cavity is investigated using TLM method. To determine the Green's functions in terms of impedance and admittance functions, a simple method is introduced. Furthermore, it is shown how to deduce equivalent circuits, which can be implemented into a commercial circuit simulator. Examples show the applicability of the technique proposed.
\end{abstract}

\section{Introduction}

In the past, for solving Maxwell's equations several approaches - among others time domain techniques are well established. In the time domain methods, space and time are discretized using different schemes. In the Transmission Line Matrix (TLM) method used here, the field space is discretized, in which the computational domain is filled with a mesh of orthogonal transmission lines. These lines periodically meet at points that are referred to as node center. As a TLM node, the local area surrounding each node center is understood. Now, the electromagnetic field in the space considered is mapped onto voltage impulses travelling on the transmission lines of the TLM mesh. The propagation properties of these voltage impulses in well-defined discrete time steps simulate the continuous phenomenon of the electromagnetic wave propagation in the physical space under investigation. The most commonly used node is the symmetrical condensed node (SCN), which is proposed by Johns [1] in 1987. Figure 1 shows a collective of such nodes. By using a system of transmission lines placed symmetrically on the branch-surface of each node, a 12-port network can be defined. The ports are situated on the cell boundaries. Figure 2 shows a pair of transmission lines at the interface of two neighboring symmetrical condensed nodes (SCNs).

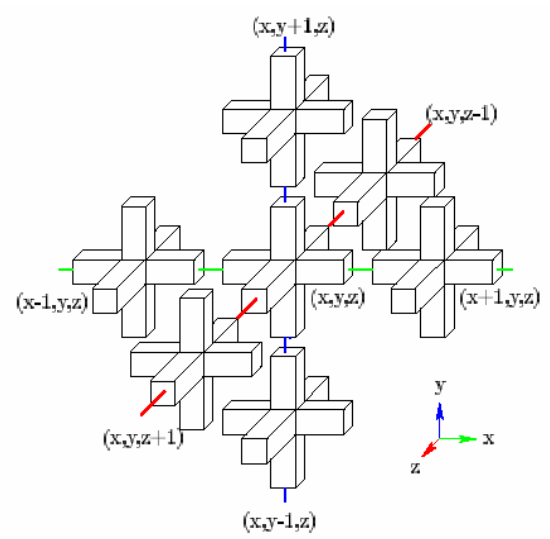

Figure 1: Part of an SCN-TLM mesh.

As depicted in Figure 2, voltage impulses travelling towards the cell boundary are designated as reflected impulses (superscript $r$ ) whereas voltage impulses travelling towards the node center are designated incident voltage impulses (superscript $i$ ).

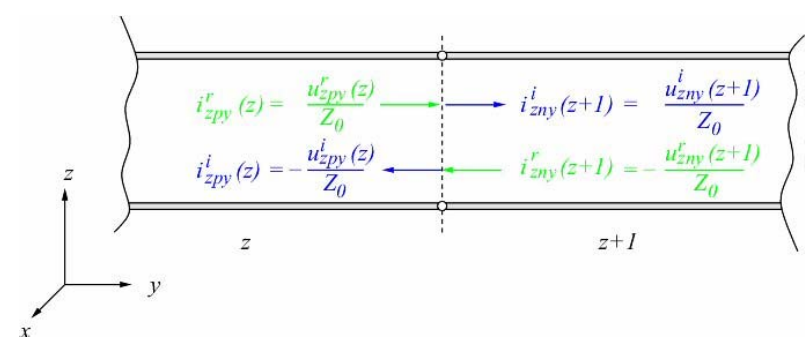

Figure 2: Interconnection of two neighboring nodes.

In the following, this interface should be investigated and cleared up, how is to find for it a suitable description using well-known tools from the theory of linear networks. 


\section{The Green's Functions in terms of Impedance or Admittance Functions}

For simplicity the deduction of equivalent circuits for the mutual coupling between cavity modes and surface current densities supported by the microstrip structure within an inhomogenously filled cavity is demonstrated for field components polarized in $\mathrm{x}$ and $\mathrm{y}$ direction as depicted in Figure 3.

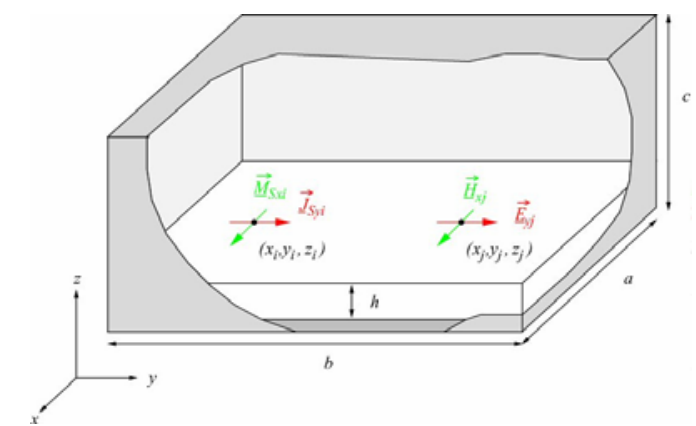

Figure 3: Geometry of a cavity under investigation.

In Figure 3, the electric dipole moment $i d l_{i}$ of the electric current density $\vec{J}_{S}$ and magnetic dipole moment $v d l_{i}$ of the magnetic current density $\vec{M}_{S}$ as well as the corresponding excited electromagnetic field components $E_{y}$ and $H_{x}$ are depicted. The relation between the dipole moments and the surface current densities are shown by equation (1) an (2):

$$
\begin{aligned}
& \vec{J}_{S}=i d l_{i} \delta\left(x-x_{i}\right) \delta\left(y-y_{i}\right) \vec{u}_{k}, \\
& \vec{M}_{S}=v d l_{i} \delta\left(x-x_{i}\right) \delta\left(y-y_{i}\right) \vec{u}_{k} .
\end{aligned}
$$

The spatial distribution of the sources are described by the delta distributions $\delta\left(x-x_{i}\right)$ and $\delta\left(y-y_{i}\right)$, respectively, whereas $\vec{u}_{k}$ is the unit vector with $\vec{u}_{k} \in\left\{\vec{u}_{x}, \vec{u}_{y}, \vec{u}_{z}\right\}$. In TLM, the implementation of the dipole sources can be accomplished by the excitation of the electric current $i$ or the voltage $v$ of the dipole moments at the interface of two adjacent cells (Figure 2). In addition, the spatial resolution in the TLM mesh is given by the cell dimensions; in this case they are: $\Delta x=\Delta y=\Delta z=\Delta l$. If the electric current $i$ excited at the boundary cell is orientated in y-direction (Figure 3), an equivalent circuit shown in Figure 4a) can be deduced.
In Figure 4a) $Z_{0}$ is the characteristic impedance of the TLM transmission lines, and $i$ current of the electric dipole moment $i d l_{i}$.

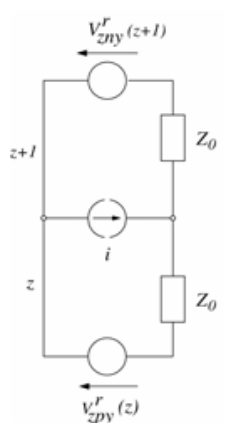

a)

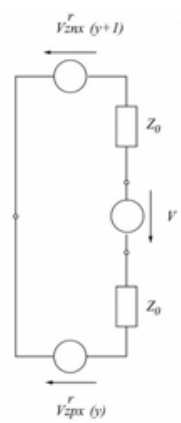

b)
Figure 4: Equivalent circuits.

a) Equivalent circuit for current excitation.

b) Equivalent circuit for voltage excitation.

Using the duality principle, the excitation of a voltage at the cell boundary can be described by the equivalent circuit drawn in Figure 4b). Alternatively the classical excitation of voltages at the cell boundary could be used. From the equivalent circuits given in Figure 4 the voltage impulses injected to the next time step at the cell boundary can be obtained by

$$
\begin{aligned}
& v_{z p y}^{i}(z)=v_{z n y}^{r}(z+1)+Z_{0} \frac{i}{2}, \\
& v_{z n y}^{i}(z+1)=v_{z p y}^{r}(z)+Z_{0} \frac{i}{2}, \\
& v_{z p y}^{i}(z)=v_{z n y}^{r}(z+1)-\frac{v}{2}, \\
& v_{z n y}^{i}(z+1)=v_{z p y}^{r}(z)+\frac{v}{2} .
\end{aligned}
$$

Beside the spatial distribution, the electric and magnetic current sources exhibit a time dependence. In TLM for the electric current $i$ and the voltage $v$ of the electric and magnetic dipole moments a modulated Gaussian impulse is used, which is given by

$a(t)=a e^{-\frac{\left(t-t_{0}\right)^{2}}{2 \sigma^{2}}} \cos \left[\omega_{0}\left(t-t_{0}\right)\right]$,

$\underline{a}(\omega)=a \sigma \sqrt{\frac{\pi}{2}} e^{-2 \pi^{2}\left(\omega-\omega_{0}\right)^{2} \sigma^{2}} e^{-j \omega t_{0}}$.

The expressions (7) and (8) are used for excitation with $a \in\{i, v\}$. It is worth a mention that expression 
(8) is the Fourier transform of a(t), and it is valid for only positive frequencies. Furthermore, with regard to Gaussian pulse excitation the Green's functions are determined in a limited frequency range.

Consider the cavity in Figure 3, which is filled by a planar dielectric layer of thickness $h$. The electromagnetic fields stimulated by a current or magnetic surface density according to equations (1) and (2) can be determined analytically using modal expansion [2]. For example, the electric field component $\underline{E}_{y j}^{m n l}$ at the observation point $\left(x_{j}, y_{j}, h\right)$ stimulated by an electric surface current density $\underline{J}_{\text {Syi }}$ at the excitation point $\left(x_{i}, y_{i}, h\right)$ can be determined as $\underline{E}_{y}^{m n l}\left(x_{j}, y_{j}, h\right)=-\underline{Z}_{m n l}$ i d l $t_{i} t_{E y m n}\left(x_{i}, y_{i}\right)$. $t_{\text {Eymn }}\left(x_{j}, y_{j}\right)$.

Herein, $t_{\text {Eymn }}$ means the y-component of the orthonormalized modal function describing the transversal electric field distribution. Furthermore, $\underline{Z}_{m n l}$ is the overall impedance seen at the location $z=h$. Using the duality principle, the corresponding magnetic field component yields:

$$
\begin{aligned}
& \underline{H}_{x}^{m n l}\left(x_{j}, y_{j}, h\right)=-\underline{Y}_{m n l} \underline{v d l_{i}} t_{\text {Hymn }}\left(x_{i}, y_{i}\right) . \\
& t_{\text {Hymn }}\left(x_{j}, y_{j}\right) .
\end{aligned}
$$

The quantities containing in expression (10) are analogous to those given in (9) and explained above.

It is well known that in the environment of a resonance frequency the impedance $\underline{Z}_{m n l}$ can be described by a shunt-tank circuit. According to the duality principle, the admittance $\underline{Y}_{m n l}$ can be realized by a series-tank circuit. Both circuits are characterized by their Laplace transforms as given below:

$$
\begin{aligned}
& \underline{Z}_{m n l}(p)=\frac{1}{C_{m n l}} \cdot \frac{p}{p^{2}+\omega_{r m n l}^{2}}, \\
& \underline{Y}_{m n l}(p)=\frac{1}{L_{m n l}} \cdot \frac{p}{p^{2}+\omega_{r m n l}^{2}}
\end{aligned}
$$

with the resonance angular frequency given by

$$
\omega_{r m n l}=\frac{1}{\sqrt{L_{m n l} C_{m n l}}}
$$

By inserting the latter expressions (11) - (13) into the field descriptions given by (9) and (10) and applying an inverse Laplace transform, the field may be obtained in time domain, too. For instance, the electromagnetic fields are to describe in terms of phase-shifted cosine functions. In lossy case, they are additionally damped.

Using the relation $\underline{E}_{y j}^{m n l}=\frac{\underline{v}_{j}^{m n l}}{d l_{i}}$ from (9), (11) and (13), the Green's functions in terms of impedance function both in the frequency and time domain can be determined

$$
\begin{aligned}
& \underline{z}_{m n l}(p)=\frac{1}{C_{m n l}} \cdot \frac{p}{p^{2}+\omega_{r m n l}^{2}} . \\
& t_{E y m n}\left(x_{i}, y_{i}\right) \cdot d l_{i} \cdot t_{E y m n}\left(x_{j}, y_{j}\right) \cdot d l_{j},
\end{aligned}
$$

$\underline{Z}_{m n l}(t)=\frac{1}{C_{m n l}} \cdot t_{E y m n}\left(x_{i}, y_{i}\right) \cdot d l_{i}$.

$t_{\text {Eymn }}\left(x_{j}, y_{j}\right) \cdot d l_{j} \cdot \cos \left(\omega_{r m n l} t\right)$.

The Green's function in terms of admittance function may be analogously obtained.

By inspection of (14), an equivalent circuit can be determined (Figure 5a). Accordingly, an equivalent circuit yields for the admittance (Figure 5b)).

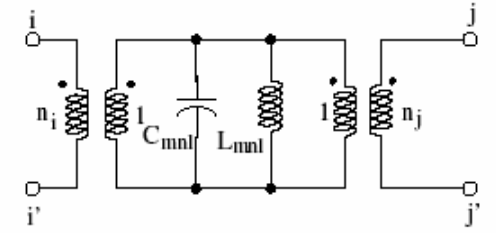

a)

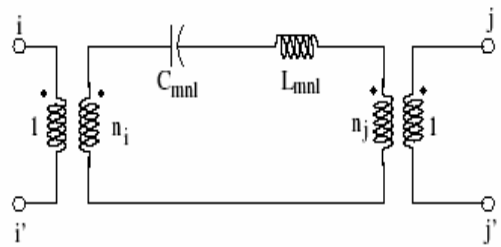

b)

Figure 5: Equivalent circuits.

a) Circuit model of the impedance function.

b) Circuit model of the admittance function.

In the equivalent circuit of Figure 5a) the turn ratios $n_{i}$ and $n_{j}$ are:

$n_{i}=t_{\text {Eymn }}\left(x_{i}, y_{i}\right) \cdot d l_{i}$,

$n_{j}=t_{\text {Eymn }}\left(x_{j}, y_{j}\right) \cdot d l_{j}$.

Using the duality principle for the admittance representation of the Green's function, similar expressions for the turn ratios $n_{i}$ and $n_{j}$ may be obtained. 


\section{Results and Discussion}

In figure 6 the dimensions of a microstip line with a shunt stub at the center of the line is shown.

(Figure 6).

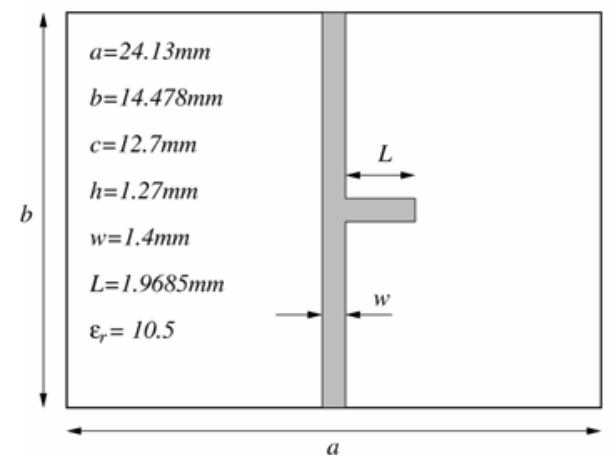

Figure 6: Geometry of a shielded microstrip line with a shunt stub.

Using the technique described above, the interaction between the microstrip structure and its package was proven by calculating the transmission response $\left|s_{21}\right|$ of this structure [3].

The results between different methods are shown in Figure 7.

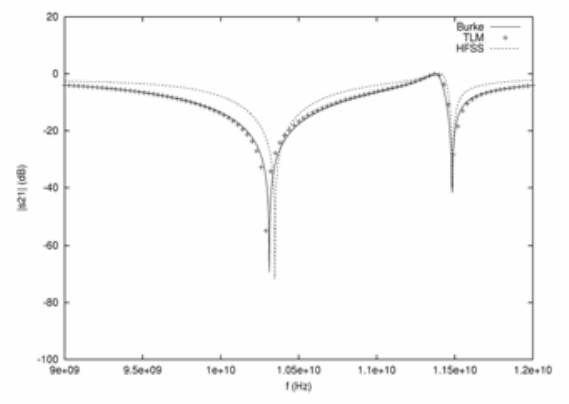

Figure 7: The magnitude of the forward transmission coefficient.

The results obtained by Burke [4] and the full wave simulator Agilent HFSS with the method introduced (Figure 7) are in good agreement.

Next, another microstrip structure was analysed, which is drawn in Figure 8 along with its dimensions. Again, the results from Burke [4] and those from Agilent HFSS are compared. As one can observe from Figure 9 , the results calculated by means of the technique proposed, are in good agreement with the independent evidences.

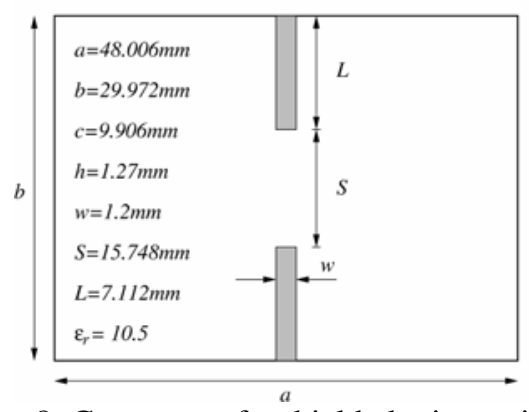

Figure 8: Geometry of a shielded microstrip gap.

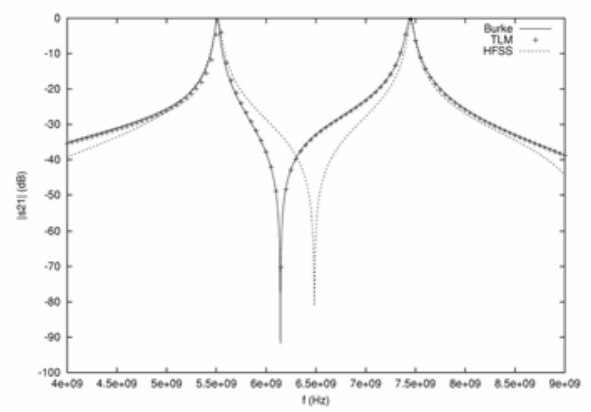

Figure 9: The magnitude of the forward transmission coefficient.

\section{Conclusion}

In this paper a technique allowing the determination of Green's functions in terms of impedance and admittance function is presented. It allows the calculation of the interaction between cavity modes and current densities supported by microstrip structures within the cavity.

\section{References}

[1] P.B. Johns, “A Symmetrical Condensed Node for the TLM-Method", IEEE Transactions on Microwave Theory and Techniques, Volume MTT-35, No. 4, April 1987, pp. 370 - 377.

[2] J.C. Rautio and R.F. Harrington, "An electromagnetic time-harmonic analysis of shielded microstrip circuits", IEEE Transactions on Microwave Theory and Techniques, Volume MTT-35, No. 8, August 1987, pp. 726 - 730.

[3] A. Sabban and K.C. Gupta, "Effects of packages on parasitic coupling among microstrip discontinuities, MMICs”, International Journal Microwave Millimeter-Wave CAE, 1(4), 1991, pp. 403 - 411.

[4] J.J. Burke and R.W. Jackson, “A simple circuit model for resonant mode coupling in packaged MMICs”, IEEE MTT-S International Symposium on MTT, Digest, Boston, MA, USA, June 1991, pp. 1121 - 1124. 